\title{
Atomistic Simulations of Thermodynamic Properties of Xe Gas Bubbles in U10Mo Fuels
}

Shenyang $\mathrm{Hu}^{*}$, Wahyu Setyawan, Vineet Joshi, and Curt.A Lavender

Pacific Northwest National Laboratory, P. O. Box 999, Richland, WA 99352

\begin{abstract}
Xe gas bubble superlattice formation is observed in irradiated uranium-10 wt\% molybdenum (U10Mo) fuels. However, the thermodynamic properties of the bubbles(the relationship among bubble size, equilibrium Xe concentration, and bubble pressure)and the mechanisms of bubble superlattice formation are not well known.In this work, the molecular dynamics (MD) method is used to study these properties and mechanisms. The results provide important inputs for quantitative mesoscale models of gas bubble evolution and fuel performance. In the MD simulations, the embedded-atom method (EAM) potential of U10Mo-Xe[1] is employed. Initial gas bubbles with a low Xeconcentration(underpressured) are generated in a body-centered cubic(bcc)U10Mo single crystal.Then Xeatoms aresequentiallyadded into the bubbles one by one, and the evolution of pressure and dislocation emission around the bubbles is analyzed. The relationship between pressure, equilibrium Xe concentration, and radiusofthe bubblesis established.It was found that an overpressuredgas bubble emits partial dislocations witha Burgers vector along the $<111>$ direction and a slip plane of (11-2). Meanwhile, dislocation loop punchout was not observed.The overpressured bubble also induces an anisotropic stress field. A tensile stress was found along $<110>$ directions around the bubble, favoring the nucleation and formation of aface-centered cubic bubble superlattice in bccU10Mo fuels.
\end{abstract}

Keywords:UMo metal fuels; Xegas bubble; thermodynamic properties; the molecular dynamics method 


\section{Introduction}

The development of low-enriched uranium fuels, such as uranium-10 wt\% molybdenum (U10Mo) dispersion fuels and monolithic $\gamma$-U10Mo fuels, is part of a global effort in support of nuclear nonproliferation [2]. Although $\gamma$-U10Mo fuels have the advantage of high density, excellent irradiation performance, and good thermal conductivity, volumetric swelling in irradiated metallic fuels is known to be an important design parameter because it affects not only the thermal conductivity of the fuels but also the mechanical integrity of fuel structures[3-5]. The fission of uranium isotopes under irradiation produces inert gas atoms such as $\mathrm{Xe}$ and $\mathrm{Kr}$ at a rate of one gas atom for every four fissionevents. These gas atoms segregate and form bubbles because of their extremely low solubility in U10Mo fuels. The fission gas bubble nucleation and growth are primarily responsible for the swelling in metallic fuels. Gas bubble structures have been examined in post-irradiation samples of dispersion and monolithic U10Mo fuels at different fission densities [2, 5-9].The intragranular bubblesare small, around 1 3 nm in diameter, and tend to form face-centered cubic (fcc)bubble superlattices[8] (i.e. it is the arrangement of the bubbles that forms an fcc not the gas atoms inside each bubble). The stability of fcc bubble superlatticestructuresin the $\gamma$-U10Mo which is a body-centered cubic (bcc) random alloy has been systematically investigated[10].However,thermodynamic properties of these bubbles,such as the relationship among bubble size, Xe concentration, and bubble pressure (i.e. the equation of states of the bubbles), and dislocation emissions from overpressured gas bubblesare not well known. A fundamental understanding of thermodynamic properties is essential for developing quantitative models of gas bubble evolution and fuel performance.

The properties of defectsin bcc U single crystals have been investigated via experiments [4, 1113], density functional theory (DFT) [14-18], and molecular dynamics (MD) method [1, 16, 19, 20].The results show that 1 ) Xe is stableas a substitutional defect, and Xe migrates through vacancy-assisted mechanisms, presumably as a complex consisting of one Xe and two vacancies ( $\mathrm{XeV}_{2}$ complex), 2) the dumbbell configurations of $U$ interstitials along the [100] or [110] direction are energetically favored, and 3 ) the migration barrier of $\mathrm{U}$ interstitials is about $0.1 \mathrm{eV}$, which is much smaller than that of vacancies $(0.5 \mathrm{eV})$. The defect generation and spatial distribution during cascades in bcc $U$ were simulated using MD methods [21, 22].The results show that most of the surviving interstitials form a [100] or [110] dumbbell.Almost all the 
interstitials remain isolated, while vacancies tend to cluster into polyhedral voids [22].The equation of state (EOS) of the Xe gas phase has been examined by experiments [23-25] and atomistic simulations [26]. Very recently, Xiao et al. simulated the pressure inside Xe bubbles with different Xe concentrations in bcc U10Mo alloys[27]. In their simulations, a bubble was introduced into the simulation cell by adding a certain number of Xe atoms into a given void. It can be imagined that the initial structure of the bubble might be far from equilibrium if the initial Xe concentration is too high. As a consequence, the matrix structure mightcollapseand unrealisticallyaffect the pressure inside the gas bubble.

In this work, we reexamine the pressure of gas bubbles in bcc U10Mo fuels more carefully using MD.In our approach, the Xe atoms are added into the bubble one by one, rather than all at once as in [26], and the system is relaxed before the next Xe is inserted. In a situation where Xe reaches the bubble mainly by vacancy-mediated migration, one may sequentially insert a $\mathrm{XeV}_{2}$ complex.In such a condition,the maximum Xe concentration is 0.5. However, underpressured and overpressured gas bubbles may exist in irradiated UMo fuels because of heterogeneous distributions of vacancies and vacancy clusters. The main goal of our work is to obtain an EOS of the bubblesthat may cover Xe concentrationlarger than 0.5. Therefore, we choose to insert Xe atom rather than $\mathrm{XeV}_{2}$. By starting with an initial concentration considerably lower than 0.5 and by relaxing the system after every insertion, insertingeither $\mathrm{Xe}$ or $\mathrm{XeV}_{2}$ would result in the same EOS, only the range of Xe concentration that can be covered is different. Based on the pressure and Xe concentration inside the bubble, a modified Van der Waals gas law and a three-parameter EOS are developed.

We note that directly adding Xe atoms into the bubble is not to rigorously mimic the gas bubble growth, but rather to achieve anoverpressured bubble and to study any interesting effects of such a bubble on the structure of the host matrix. Indeed, the growth mechanism of gas bubbles is complex since the dynamics of defect diffusion and displacement cascade would play a role.Nevertheless, for a given bubble condition (size and Xe concentration), an EOScan be constructed. In other words, the results of our study are valid for a situation where the bubbles have enough time to relax before any changes of Xe concentration occur (e.g. due to Xe diffusion or cascade effect). While it is not the main purpose of this paper to explain the formation of bubble superlattice, we will discuss how the results of our study may improve the understanding of gas bubble superlattice formation. To this end three plausible mechanisms were proposed to explain the gas bubble superlattice formation; elastic interaction, one-dimensional migration of self-interstitial atoms (SIAs), and dislocation punch out[8, 28, 29]. Our recent work [30] explored the first two mechanisms. It was found that the one-dimensional migration of SIAs along $<110>$ directions is the dominant mechanism for the fcc gas bubble superlattice formation in bcc UMo fuels. In this paper, by monitoring the evolution of the bubbles and matrix atoms as 
a function of bubble size, we will explore whether the overpressured bubble will emit dislocation loops or not and gain understanding of the third mechanism (i.e. the dislocation loop punch out).

\section{Simulation Methodology}

\subsection{Interatomic Potential}

Recently,Smirnova et al. developed anembedded-atom method (EAM) potential for U10Mo-Xe systemsusing a force matching technique and a dataset of ab initio atomic forces[1]. The EAM potential has been used to assess the general properties of the U, Mo, Xe, U-Mo alloys, and the $\mathrm{U}_{2}$ Mo intermetallic[1]. The predicted lattice constants, elasticconstants, thermal expansion, and melting temperature of all thephases are consistent with the experimental data. The simulations proved that the $\gamma$-U10Mo structure built with U-Mo-Xe potential remains stable in the temperature range from $300 \mathrm{~K}$ up to the melting temperature ( 1500K) [1]. The self-diffusion coefficients in pure $\gamma$-U and $\gamma$-U10Mo alloys have also been calculated using MD with the EAM potential[31]. The results show that self-interstitial atoms play a leading role in the self-diffusion

processes, and explain well theremarkably high self-diffusion mobility observed experimentally for $\gamma-\mathrm{U}$ [31]. In addition, the EOS of the Xe gas phase predicted using the EAM potential agrees well with experimental data in a broad range of temperatures and pressures[26]. Therefore, the EAM potential is sufficiently reliable,because it can reproduce the lattice constants, elasticconstants, thermal expansion, and EOS as well as the defect properties.In this work, the EAM potential was used to study the evolution of pressure and dislocation emission with an increase of Xe concentration inside the intragranular gas bubbles in bcc U10Mo alloys.

\subsection{MD simulation process}

IntragranularXe gas bubbles often form a superlattice inside large grains in irradiated U10Mo fuels. To study the potential effect of gas bubble structures on the thermodynamic property (i.e. the EOS) of gas bubbles, we consider two gas bubble superlattices; simple cubic (sc) and fcc lattices, which are shown in Figure 1. In anscsuperlattice, the bubble can be viewed as an isolated one if there is no interaction between the bubbles,i.e., when the superlattice constant is large enough.For the fccsuperlattice, bubbles with a superlattice constantof 10 20nm are set up to mimic the bubble superlattice observed in experiments. Bubbles with an initial radius of $1 \sim 3$ 
$\mathrm{nm}$ are explored. The MD program LAMMPS[32]is employed to simulate the evolution of thermodynamic properties of intragranular gas bubbles, including pressure, Xe concentration, and dislocation emission. The temperature is controlled using a Nose'-Hoover thermostat [33] while thepressure is controlled using a Nose'-Hoover barostat[34].The time step for all of the MD simulationsis $1 \mathrm{fs}$.

(Insert Figure 1. here)

Figure 1.(a) gas bubbles with anscsuperlattice, and (b) gas bubbles with an fccsuperlattice. The gas bubble superlattice constant, a, is shown in Figure 1b. The superlattices are simulated within a bcc U10Mo crystal. The blue, green, and gray balls represent U, Mo, and Xe atoms, respectively.

The MD simulation process is described as follows:

1) An equilibrium configuration of bcc U10Mo single crystal is obtained with MD simulations at constant temperature of $500 \mathrm{~K}$ and zero pressure.

2) One or four gas bubbles, depending on the gas bubble superlattice structure,are introduced into the single crystal by removing the matrix atoms ( $\mathrm{U}$ and Mo) and adding certain Xe atoms, as shown in Figure 1. The gas bubble has an initial radius determined by the spherical void where the matrix atoms ( $\mathrm{U}$ and Mo) are removed. The initial Xe concentration inside the gas bubble is defined as the ratio between the number of added Xe atoms and the number of vacancies inside the spherical voids. The gas bubble superlattice has a lattice constant "a” shown in Figure 1b. 3) Xe atomsareinserted into the gas bubble one by one. After every insertion, the system is relaxed for 10 pswith constant volume and temperature (NVT)before the next atom is inserted. 4) Thermodynamic properties including the average radius of the gas bubble, average Xe concentration, and pressure inside the gas bubble are calculated for a relaxed atomic configuration within a 10ps period. The characterization of defects is carried out using Open Visualization Tool (OVITO) software[35]. Stacking faults are determined by using common 
neighbor analysis (CNA) [36] and the centro-symmetry parameters (CSP) [37], and the evolutions of the dislocation lengths and densities are quantified using the dislocation extraction algorithm(DXA)[35, 38].

The components of the atomic-level stress tensor are calculated as shown in equation (1):

$$
\sigma_{\alpha \beta}(i)=-\frac{1}{\Omega_{0}}\left[\frac{1}{2} \sum_{j} F_{i j}^{\alpha} r_{i j}^{\beta}+M_{i} V_{i}^{\alpha} V_{i}^{\beta}\right]
$$

where $\alpha$ and $\beta$ label the Cartesian components, $\Omega_{0}$ is the average atomic volume, $F_{i j}$ is the force on atom $i$ due to atom $j, r_{i j}$ is the vector coordinate of neighbor atom $j$ relative to atom $i, M_{i}$ is the mass of atom $i$, and $V_{i}$ is the velocity of atom $i$. The pressure on atom $i$ is calculated as $P(i)=-\frac{1}{3}\left(\sigma_{x x}(i)+\sigma_{y y}(i)+\sigma_{z z}(i)\right)$, where $\sigma_{x x}, \sigma_{y y}$, and $\sigma_{z z}$ are the stress components in the $x$, $y$, and $z$ directions, respectively.

\section{EOS of Xeintragranular gas bubbles}

We first examine the gas bubble evolution where the bubblesform anscsuperlattice with a lattice constant ( $\left.a=46 a_{0}=16 \mathrm{~nm}\right)$, an initial radius $\left(R_{0}=0.8 \mathrm{~nm}\right)$, and initial Xe concentration ( $C_{X e}^{0}=0.3$ ). $a_{0}$ is the lattice constant of the bcc U10Mo. The evolution of theatom configuration in the slice A shown in Figure 1a is presented in Figure 2. An analysis of common neighbors using OVITO shows that the majority of the host lattice (more than 95\%)remains in bcc structure at $500 \mathrm{~K}$ although there is a certain lattice distortion in U10Mo. The evolution of the gas bubble morphology clearly indicates that the bubble size and the local lattice distortion and/or deformation around the bubble increasesas the number of Xe atoms $\left(N_{X e}\right)$ inside the bubble increases. Growth of a gas bubble requires a continuous supply of vacancies and gas atoms. The vacancies could come from the bulk vacancy diffusion or be generated via displacement ofU and Mo atomsfrom the lattice or via dislocation emission. Since bulk vacancy diffusion is highly unlikely in MD simulation at low temperature during a short simulation time, the required 
vacancies for the bubble growth should be generated viadislocation emission. More detailed defect analysis associated with bubble growth will be given in section 4 .

(Insert Figure 2. here)

Figure 2. Temporal evolution of atomic configuration around the gas bubble on the slice A shown in Figure 1a. The blue, green, and gray particles denote U, Mo, and Xe atoms, respectively. The slice thickness is five atomic layers with the normal direction [110]. $N_{X e}$ is the total number ofXe atoms inside the gas bubble.

The changes of bubble radius, Xe concentration, and bubble pressureare calculated. The bubble radius is definedas $R=\left(R_{\text {outer }}+R_{\text {inner }}\right) / 2$ where $R_{\text {outer }}$ and $R_{\text {inner }}$ are the radii of bubble's outerand inner shells, respectively. $R_{\text {outer }}$ is the distance of the farthest Xe atom in the bubble from the bubble center, while $R_{\text {inner }}$ is the distance of the closest $\mathrm{U}$ or Mo atom in the matrix from the bubble center. The bubble center is the center of mass of the Xe atoms in each bubble. The Xe concentration is calculated as $C_{X e}=N_{X e} / N_{V a c}$, where $N_{X e}$ and $N_{V a c}$ are the total numbers of Xe atoms and vacancies inside the gas bubble, respectively. The vacancy number $N_{\text {Vac. }}$ is calculated as $V / \Omega_{0}$, where $V$ is the volume of the gas bubble $\left(V=4 \pi R^{3} / 3\right)$ and $\Omega_{0}$ is the average atom volume in bcc U10Mo alloys. The average pressure is defined as $\bar{P}=-\sum_{i=1}^{N_{X e}}\left(\sigma_{x x}(i)+\sigma_{y y}(i)+\sigma_{z z}(i)\right) / 3$. Figure 3 shows the temporal evolution of pressure,Xe concentration, and gas bubble radius as a function of $N_{X e}$ for bubbleswith different initial radii $R_{0}$. The pressure, Xe concentration, and bubble radius increase as $N_{X e}$ increases. The pressure of a bubble with a small initial radius $R_{0}=0.8$ nmincreasesmore rapidly than that ofa bubble with a large initial radius $R_{0}=1.0 \mathrm{~nm}$, which is understandable because there is more free space inside the larger bubble if the bubbles have the same initial Xe concentration.It is interesting that the bubble pressure changes non-monotonically,as shown by the zig-zag pressure curves.However, 
the pressure evolution of the small bubble is quite different from that of the large bubble. For example, the first pressure drop occurs at 8GPa for the bubble with $R_{0}=0.8 \mathrm{~nm}$, while it occurs at 6GPa for the bubble with $R_{0}=1.0 \mathrm{~nm}$. The pressure drop is larger for the small bubble than for the large bubble.

Examining the atomic configuration, we find that the large pressure drop from $N_{X e}=83$ to $N_{X e}=$ 113shown in Figure 2 is associated with partial dislocation emission and deformation twinning. As we know, for the same internal pressure,the stresses around a small bubble are smaller than those around a large bubble. Therefore, it is understandable that a larger internal pressure inside a smaller bubble is required to reach the critical stress for dislocation nucleation. The larger pressure drop in a smaller bubble can be explained by the same amount of vacancy generation during dislocation nucleation and emission.The pressure inside the bubble with $R_{0}=0.8$ nmdecreasesas the size increases. It is consistent with the Van der Waals gas law that the pressure decreases with the increase of gas bubble size if the interface energy is constant.The pressure inside the bubble with $R_{0}=1.0 \mathrm{~nm}$ increases slightlywith size. It can be seen that, regardless of the initial size,the pressuresof the small and large bubbles converge once the number of Xeatoms reaches about 225 (a bubble radius of about $1.2 \mathrm{~nm}$ ). When the gas bubble radius is larger than $1.2 \mathrm{~nm}$ (up to the largest radius of $1.5 \mathrm{~nm}$ that the simulations reach), the pressure and Xe concentration are almost constants, namely at about 6.05 6.35GPaand $0.49 \sim 0.50$, respectively.

(Insert Figure 3. here)

Figure 3.Predicted gas bubble radius, pressure, and Xe concentration inside the bubble as a function ofthe number of Xe atoms $N_{X e}$ inside the bubble.

The pressure inside a Xe gas bubble in U10Mo alloys was previously simulated by Xiao et al.[27]using the same EAM potential[1]. The parameters used in their simulations, such as temperature $\mathrm{T}=500 \mathrm{~K}$, initial void $\operatorname{size} R_{0}=0.8 \mathrm{~nm}$, MD time step $1 \mathrm{fs}$, and atomic potential, are 
the same as those for the case with small initial gas bubble radius in our simulations. However, they added different amounts of Xe atoms into a void at the initial stage, while we added the Xe atoms into the bubble one by one followed by 10 ps of equilibration under constant volume and temperature (NVT). In addition, they used a periodic cubic supercell $\left(16 a_{0} \times 16 a_{0} \times 16 a_{0}\right)$ that is much smaller than our simulation cell ( $\left.46 a_{0} \times 46 a_{0} \times 46 a_{0}\right)$. Comparing the results, we found some similar phenomena:1) a pressure drop takes place between $N_{X e}=80$ and $N_{X e}=90$, and 2) the pressure for a bubble with a radius around 1.0 1.2 nm isalmostconstant. The pressure drop in their simulations is about 7GPa, which is much larger than the 2GPaobserved in our simulations. The value of pressure after it becomes constant is about 4GPa, which is lower than the 6GPa predicted in our simulations.As we can imagine, an unphysically high initial Xe concentration inside a void may drive the system far away from equilibrium. As a result, an unrealisticallylarge lattice distortion and amorphization around the bubble may happen, which would affect the pressure inside the bubble. Figure 2 shows the dislocation emission and deformation twinning that are observed in our simulations, while they are not observed around the overpressured gas bubble in their simulations.Therefore, the overestimated pressure drop and underestimated equilibrium pressure in Ref [27]are likely the consequences of an unrealistic MD simulation process.

To examine the effect of fcc gas bubble superlattice structure on pressure and Xe concentration, anfcc gas bubble superlatticewith a similar bubble size (1 3 nmin diameter) and superlattice constant ( $a=46 a_{0}=16 \mathrm{~nm}$ ) as observed in irradiated U10Mo fuels was considered. The same simulation process described above was employed.The results are plotted in Figure 4together with the results from the scsuperlattice simulations for comparison. It can be seen that the bubble pressure evolution and the relationships among pressure, Xe concentration, and gas bubble diameters for the sc and fccbubble superlattices are very similar. Note that, in this fixed volume (constrained) simulation, the overall pressure on the simulation cell with fcc bubble superlattice is larger than that of the sc bubble superlattice. However, this difference is only in the MPa regime, which is negligible compared to the internal pressure of the bubble which is in the GPa regime. For instance,the overall pressure on the simulation cell with the fcc bubble superlattice changes from 0toonly $12 \mathrm{MPa}$ with the increase of $N_{X e}$ while it changes from 0to only 3.8MPa with the sc bubble superlattice. The overall pressure difference can be explained by the facts that 
1) our simulations were conducted under constant volume and temperature, and 2) the gas bubble density withthe fccsuperlattice is four times that withthe scsuperlattice, because bothsuperlattices have the same lattice constant $a=46 a_{0}$.The results indicate that the equilibrium pressure and Xe concentration inside the gas bubble will increase as the gas bubble density increases.

(Insert Figure 4. here)

Figure 4.(a) Predicted relationship between pressure and Xe concentration (i.e. the EOS) for sc and fccbubble superlattices; (b) Predicted relationship between pressure and gas bubble size.

In a rate theory, the EOS of intragranular gas bubbles is often described by a modified Van der Waals gas law[39],

$$
\left(\frac{2 \gamma}{R}+P_{0}\right)\left(\frac{4 \pi R^{3}}{3}-h N_{X e}\right)=N_{X e} k T
$$

where $\gamma$ is the interfacial energy, $P_{0}$ is the hydrostatic pressure, $N_{X e}$ is the total number of Xe atoms inside the gas bubble, $k$ is Boltzmann's constant, $T$ is the absolute temperature, and $h$ is a fitting parameter. The interfacial energy $\gamma$ is usually set to be about $0.5 \mathrm{~J} / \mathrm{m}^{2}$ in rate theory models of Xe gas bubble evolution in U10Mo alloys[39]. Compared with the pressure (a few GPa) inside the gas bubble, the hydrostatic pressure $P_{0}$ (the overall pressure on the simulation cell) is low (about a few MPa), and therefore can be neglected. With the assumption $P_{0}=0$, the parameter $h$ can be fitted using the relationship amongXeconcentration $C_{X e}=N_{X e} \Omega_{0} /\left(4 \pi R^{3} / 3\right)$, $N_{X e}$, and $R$ as shown in Figure 4.The fitted parameter is $h=1.15 \times 10^{-28}$. Figure 5 plots the total number of Xeatoms inside a gas bubble in MD simulations versus thatcalculated using equation (2). The symbolsrepresent the results of four simulation cases with different initial bubble sizes and different bubble superlattices. The red dashed line indicates the diagonal of the chart with a slope $=1$. If the simulation curve follows the dashed line, it means that the total number of Xe atoms from MD simulations is equal to thatpredicted using equation (2). It can be seen that the 
majority of the data falls on the diagonal line.In other words, the EOS of a Xeintragranular gas bubble in U10Mo alloys at 500K can be well described by the modified Van der Waals gas law equation (2) with $\gamma=0.5 \mathrm{~J} / \mathrm{m}^{2}$ and $h=1.15 \times 10^{-28}$.

\section{(Insert Figure 5. here)}

Figure 5.(a) Xe concentration from MD versus that calculated using equation (2); (b) temporal evolution of pressure and Xe concentration inside the gas bubble.

Regarding the EOS (the relationship between pressure and molar volume of Xe gas phases), a three-parameter EOS is proposedby Kaplun and Meshalkin[40]. It is written as

$$
P=\frac{R T}{V}\left(1+\frac{a}{V-b}\right)-\frac{c}{V^{2}}
$$

where $P$ is the pressure, $V$ is the molar volume, and $a, b$, and $c$ are fitting parameters. This equation is also used to fit the EOS of the Xe gas phase inside the gas bubble. Fitting equation (3) with the data in Figure 4 and the relationship $V=\frac{4 \pi R^{3} N}{3 N_{x}}$, where $N$ is the Avogadro constant, yields $a=18.928 \mathrm{~cm}^{3} / \mathrm{mol}, b=259,780 \mathrm{Jcm}^{3} / \mathrm{mol}^{2}$, and $c=280.658 \mathrm{~cm}^{3} / \mathrm{mol}$. The relationship between $P$ and $V$ obtained from MD simulations and fitted equation (3) is presented in Figure 5b.The symbols show the results from MD simulation, while the solid line shows the fitted equation. We can see that the three-parameter EOS can describe well the relationship between the pressure and molar volume of the Xe gas phase inside intragranular gas bubbles.

We note that constrained simulations under NVT perhaps better represent the condition in monolithic fuels given that the UMo foil is embedded in anAl cladding. We also note that, as previously described, the external pressure acting on the simulation cell with sc and fcc gas bubble superlatticesis much smaller (in the order of MPa) compared to the internal bubble pressure (in the order of GPa). Nevertheless, to examine the effect of the constraint NVT 
conditions on the EOS and possible dislocation emission from the over pressured gas bubbles, the evolution of gas bubbles in sc and fcc gas bubble superlattices with initial radius $R_{0}=1.0 \mathrm{~nm}$ and Xe concentration ( $C_{X e}^{0}=0.3$ ) were additionally simulated under NPT in a simulation cell ( $46 a_{0} \times 46 a_{0} \times 46 a_{0}$ ). The simulations under NPT were performed with zero external pressure and represent unconstrained conditions. The results obtained under NVT and NPT are plotted in Figure 6.From Figure 6a, we can see that the EOS (i.e. the relationship between the pressure and Xe concentration inside the gas bubbles, not between pressure and bubble diameter) is independent of the simulation condition (NVT or NPT), i.e. the EOS with NVT and NPT superimposes each other. The EOS also does not depend on the superlattice (sc or fcc). While the EOS is invariant, the NVT condition allows for a higher pressure to build up then NPT as expected.

The larger maximum pressure that can be reached with NVT can be more clearly seen in Figure $6 \mathrm{~b}$ where thebubble pressure is plotted as a function of the gas bubble diameter (note that the relationship between pressure and diameter is not an EOS). Before the pressure reaches 6GPa, the pressure obtained under NVT and NPT are practically the same. Beyond 6GPa, it is observed that 1) the pressure varies up and down which is associated with the dislocation emission, and 2) the average pressure simulated under NVT is larger than that simulated under NPT, particularly for the fccsuperlattice. Dislocation emission will release the gas bubble pressure. The high gas bubble pressure under NVT implies that critical stress for dislocation emission under NVT is larger than that under NPT. Itwill be discussed in next sections that while the critical stress is different, the mechanism of the dislocation emission is the same under NVT or NPT.

(Insert Figure 6. here)

Figure 6. Comparison of the results obtained under NVT and NPT. (a) Pressure as a function of Xe concentration inside the gas bubble (i.e. the EOS), (b) Pressure as a function of the gas bubble size.

\section{Dislocation emission around overpressured gas bubbles}


Dislocation punchout from an overpressurized gas bubble was proposed as a mechanism of bubble growth and bubble ordering[28, 41-43]. In bcc crystals,an overpressurized bubble may punch out prismatic loops that lie in either $\{111\}$ or $\{110\}$ habit planes with a Burgers vector $1 / 2<111>[28]$. Figure 7 shows snapshots of the atomic configuration on (110) planes around the gas bubble with a scsuperlattice. The atoms on two (110) atomic layers are presented. The white circles denote the atoms on the top layer while the gray circles denote the atoms on the bottom layer. In Figure 7a, it can be seen that there are local lattice distortions marked by the red ellipses However, the analysis of common neighbors using OVITO shows that more than $95 \%$ of the lattices in the matrix retain the bcc structure. The large lattice distortion in bcc U10Mo is also observed in DFT calculations[30]. The dislocation analysis using OVITO's DXA indicates there are only a few dislocation segments emitted from the gas bubble duringthe increase of $N_{\text {Xe }}$ from 78 to 377 . The dislocation segment has a Burgers vector of a/2<111 $>$ and $\{112\}$ slip planes. However, Figures7b-d showthat partial dislocations withBurgers vectorsalong $<111>$ and $\{112\}$ slip planes are continuously emitted from the gas bubble as $N_{X e}$ increases. The partial dislocation emission formsa distribution of stacking faults and nano-twin-like structures near the gas bubble.Most dislocations localize inside the diagonal channels of the cubic simulation cell due to the anisotropy of the resolved shear stresses on the slip planes. As a result, a star-shaped dislocation structure is formed around the gas bubble. In addition, it is interesting to find that the interface of the gas bubble gradually becomes faceted with the continuous emission of dislocations. The faceted interface is the plane $\{111\}$. This implies that the gas bubble has a faceted shape. Experimentsclaimed that Xegas bubbles have faceted shape[8].The simulation results suggest that the evolution of dislocation structure around the gas bubble may be one of the mechanisms for the faceted gas bubble formation. The punching out of $1 / 2<111>[111]$ dislocation loops will form extraatomic layers on (111) habit planes. Examining the atomic configuration, we find there are noextraatomic layers on habit planes $\{111\}$ or $\{110\}$. In other words, the overpressurizedXegas bubble in U10Mo alloys doesnot punchout dislocation loops, but rather emits only partial dislocations.

(Insert Figure 7. here) 
Figure 7.Evolution of atomic configuration, partial dislocations, and nano-twin structures around the gas bubble as a functionof $N_{X e}$.

Figure 7d shows that the lattice distortion fields around the bubble do not overlap. This implies that the gas bubble in the scsuperlattice could be viewed as an isolated bubble. In other words, the characteristics of dislocation emission observed in the scsuperlatticecorresponds to that of anisolated gas bubble. In irradiated U10Mo fuels, the gas bubbles form fccsuperlattices. To study the potential effect of gas bubble structureson dislocation emission, anfcc gas bubble superlatticewith similar bubble size and lattice constant as observed in irradiated U10Mois considered.The initial bubble size and Xe content insc and fcc bubble superlattices are the same. Figure 8 shows the atomic configuration on the (110) plane forthe gas bubbles shown in Figure 1b. Each bubble contains $N_{X e}=342$. Stacking faults and twin-like structures around the bubbles are observed in Figure 8 (for the fcc bubble superlattice), similar to those observed in Figure 7d (for the isolated bubble). Therefore, we can conclude that for both isolated gas bubbles and gas bubbles arranged in fccsuperlattices, partial dislocation emission is observed.

(Insert Figure 8. here)

Figure 8. Atomic configuration on the (110) plane for the fccbubble superlattice shown in Figure $1 b$.

\section{Evolution of stress field around the gas bubble}

A continuous emission of partial dislocations from gas bubbles may causedislocation pileup and generate aninhomogeneous stress field around the bubbles. The local stress state affects the bubble nucleation, and hence the bubble structures. For instance, a tensile stress will be favorable for the nucleation and growth of gas bubbles. We analyze the distribution of the average atom density and pressure on the lines passing through the bubble center along $<110>$ and $<111>$ directions.The atom density and pressure at point $r$ are defined as the respective averagesfor all 
the atoms inside a sphere with a radius $r_{0}$ and the center at $r$.In this work, $r_{0}$ is set to be $7 \mathrm{~nm}$, which is slightly larger than the second-nearest-neighbor distance in bcc U10Mo. The average atom density of $\mathrm{U} 10 \mathrm{Mo}$ at $500 \mathrm{~K}$ is set to be 1 as a reference state. Then,the change ofthe average atom density can be calculated as

$$
\Delta C(r)=\frac{m}{V_{0} / \Omega_{0}}-1
$$

where $m$ is the number of atoms inside the spherecentered at $r, V_{0}=4 \pi r_{0}^{3} / 3$ is the volume of the sphere, and $\Omega_{0}=1.983 \times 10^{-29} \mathrm{~m}^{3}$ is the average atom volume of bcc U10Mo alloys at $500 \mathrm{~K}$, which is predicted by MD with the EAM potential. There are two lines passing through the bubble center with $<110>$ direction and four lines with $<111>$ direction. The change of the average atom density $\Delta C(r)$ along these six lineswas calculated atfour bubble states $\left(N_{X e}=78\right.$, 177, 277, and 377). The results are plotted in Figure 9. The dashed lines $(\Delta C(r)=0)$ indicate the reference state.Inside the gas bubble, the Xe concentration, calculated using $1+\Delta C(r)$, reaches 0.5 as $N_{X e}$ increases, which is consistent with the results shown in Figure 3. There is a regionjust outside the bubble-matrix interface where $\Delta C(r)$ is negative. That means that this region is less dense than the reference matrix.Outside this region, the atom density along $<110>$ directions is almost constant and similar to that of the reference state. However, along $<111>$ directions, the distributions of atom density arenot uniform. The arrow in Figure 9b indicates that a peak of $\Delta C(r)$ moves away from the gas bubble as $N_{X e}$ increases.As we know, at the core of an edge dislocation,the atom density increases above the slip planewhile it decreases below the slip plane. So the nonuniform atom density along the $<111>$ direction indicates a nonuniform dislocation distribution. The shift of the $\Delta C(r)$ peaks corresponds to the expansion of the partial dislocations and nano-twin structures shown in Figure 7.

(Insert Figure 9. here)

Figure 9.Change of the average atom density on lines passing through the gas bubble center (a) along the $<110>$ direction, and (b) along the $<111>$ direction. 
The atomic-level stresses can be calculated using equation (1). The average pressure $\bar{P}(r)$ inside the sphere is definedby equation (5):

$$
\bar{P}(r)=\sum_{i=1}^{m}\left[\frac{1}{3}\left(\sigma_{x x}(i)+\sigma_{y y}(i)+\sigma_{z z}(i)\right)\right] / m
$$

For the gas bubble with $N_{X e}=377$, the distributions of the average pressures on the two lines along the $<110>$ direction and the four lines alongthe $<111>$ direction are plotted in Figure 10a and 9b, respectively.According to the definition of $\bar{P}(r)$ in equation (5), a positive value means a tensile stress while a negative value means a compressive stress. The pressure inside the gas bubble is almost uniform at about 5 GPa. However, the atomic-level stresses in the matrix show a large variation. Two main factors may cause this large variation of the atomic-level stresses. One is that the local variation of Mo distribution U10Mo may cause a nonuniform atomic-level stress. The other factor is the partial dislocations emitted from the gas bubble. The stress changes from tensile to compressive across the slip plane at a dislocation core. So a distributed dislocation will cause a large variation of stress. However, the result shows that the majority of atoms on the line with $<110>$ direction have tensile stresses, and the majority of atoms on the line with $<111>$ direction have compressive stresses. Such a stress field around the gas bubble may result in the favorable nucleation of gas bubblesalongthe $<110>$ direction. If gas bubbles prefer to nucleate along the $<110>$ directions in bcc crystals, the gas bubbles will form an fccsuperlattice. Therefore, the stress-induced nucleation of gas bubbles on the $<110>$ directioncould be one factor in the formation of the fccXe gas bubble superlatticeobserved in the experiments.

(Insert Figure 10. here)

Figure 10. Distribution of the average pressure (a) along the $<110>$ direction and (b) along the $<111>$ direction for $N_{X e}=377$. 
Recently, a phase-field model of gas bubble evolution in irradiated U10Mo fuels was developed to study the mechanism of gas bubble superlattice formation[30]. The results suggested that 1) elastic interaction does not play an important role in the fccsuperlattice formation, and the dominant mechanism is instead the fast one-dimensional migration of interstitials. In the phasefield model, it is assumed that 1) the deformation obeys linear elasticity,and 2) the equilibrium pressure inside the gas bubble is about 2GPa. However, our atomistic simulations show that even under an unconstrained condition (NPT), the equilibrium pressure of the gas bubbles is about 56GPa, considerably higher than that used in the phase-field model. If excess Xe atoms reach a bubble in equilibrium pressure, the bubble will emit partial dislocations to maintain the equilibrium pressure. Thissuggests that plastic deformation, which may change the stress field around the gas bubble, is important for modeling Xegas bubble growth. It is important to note that while the EOS that is developed in this study is valid in terms of describing the pressure of a bubble for a given Xe concentration, it does not tell us whether bubbles in irradiated U10Moare actually underpressured, in equilibrium pressure, or overpressured. Therefore, accurate atomistic simulations can provide more reliable thermodynamic data and mechanisms of microstructure evolution for more quantitative mesoscale phase-field modeling of gas bubble evolution.

\section{Conclusions}

In this work, MD simulations are performed to study the thermodynamic properties of Xe gas bubbles in bcc U10Mo alloys. Using the calculated pressure, Xe concentration, and gas bubble radius, we develop a three-parameter EOS to describe the relationship between the pressure and molar volume of the Xe gas phase inside the bubble, and employ a modified Van der Waals gas law to describe the relationship between bubble radius and Xe content inside the bubble at $500 \mathrm{~K}$ (close to the operation temperature of monolithic U10Mo fuels). The evolution of the atomistic configuration shows partial dislocation emissions from overpressured gas bubbles to maintain an equilibrium pressure. The emitted partial dislocations have a $<111>$ Burgers vector and a (11-2) slip plane. Dislocation loop punchout isnot observed. A tensile stress is found along $<110>$ directions around the bubble,favoring the nucleation and formation of an fcc bubble superlattice in bcc U10Mo fuels. The developed EOS and improved understanding of gas bubble 
growth mechanisms are important inputsfor quantitativemesoscale modeling of gas bubble evolution and swelling in irradiated U10Mo fuels.

\section{Acknowledgements}

The work described in this article was performed by Pacific Northwest National Laboratory, which is operated by Battelle for the United States Department of Energy under Contract DEAC05-76RL01830. This study was supported by the U.S. Department of Energy, National Nuclear Security Administration Office of Material Management and Minimization Reactor Conversion Program. 


\section{References}

[1] D.E. Smirnova, A.Y. Kuksin, S.V. Starikov, V.V. Stegailov, Z. Insepov, J. Rest, A.M. Yacout, Model Simul Mater Sc 21 (2013) 1-24.

[2] Y.S. Kim, G.L. Hofman, J Nucl Mater 419 (2011) 291-301.

[3] Y.S. Kim, G.L. Hofman, J.S. Cheon, A.B. Robinson, D.M. Wachs, J Nucl Mater 437 (2013) 37-46.

[4] R.M. Hengstler, L. Beck, H. Breitkreutz, C. Jarousse, R. Jungwirth, W. Petry, W. Schmid, J. Schneider, N. Wieschalla, J Nucl Mater 402 (2010) 74-80.

[5] Y.S. Kim, G.L. Hofman, J.S. Cheon, J Nucl Mater 436 (2013) 14-22.

[6] J. Gan, D.D. Keiser, B.D. Miller, A.B. Robinson, J.F. Jue, P. Medvedev, D.M. Wachs, J Nucl Mater 424 (2012) 43-50.

[7] D.D. Keiser, J.F. Jue, A.B. Robinson, P. Medvedev, J. Gan, B.D. Miller, D.M. Wachs, G.A. Moore, C.R. Clark, M.K. Meyer, M.R. Finlay, J Nucl Mater 425 (2012) 156-172.

[8] B.D. Miller, J. Gan, J.D.D. Keiser, A.B. Robinson, J.F. Jue, J.W. Madden, P.G. Medvedev, J Nucl Mater 458 (2015) 115-121.

[9] S. Van den Berghe, W. Van Renterghem, A. Leenaers, J Nucl Mater 375 (2008) 340-346.

[10] J. Gan, D.D. Keiser, B.D. Miller, A.B. Robinson, D.M. Wachs, M.K. Meyer, J Nucl Mater 464 (2015) 1-5.

[11] D.E. Burkes, C.A. Papesch, A.P. Maddison, T. Hartmann, F.J. Rice, J Nucl Mater 403 (2010) 160166.

[12] K. Huang, D.D. Keiser, Y.H. Sohn, Metall Mater Trans A 44A (2013) 738-746.

[13] S.J. Rothman, L.T. Lloyd, A.L. Harkness, T Am I Min Met Eng 218 (1960) 605-607.

[14] B. Beeler, B. Good, S. Rashkeev, C. Deo, M. Baskes, M. Okuniewski, J Nucl Mater 425 (2012) 2-7.

[15] B. Beeler, B. Good, S. Rashkeev, C. Deo, M. Baskes, M. Okuniewski, J Phys-Condens Mat 22 (2010) 1-7.

[16] B. Beeler, C. Deo, M. Baskes, M. Okuniewski, J Phys-Condens Mat 24 (2012) 1-9.

[17] B. Beeler, C. Deo, M. Baskes, M. Okuniewski, J Nucl Mater 433 (2013) 143-151.

[18] A. Landa, P. Soderlind, P.E.A. Turchi, J Nucl Mater 414 (2011) 132-137.

[19] D.E. Smirnova, S.V. Starikov, V.V. Stegailov, J Phys-Condens Mat 24 (2012) 1-.

[20] D.E. Smirnova, A.Y. Kuksin, S.V. Starikov, V.V. Stegailov, Phys Met Metallogr+ 116 (2015) 445-455.

[21] X.F. Tian, H.X. Xiao, R. Tang, C.H. Lu, Nucl Instrum Meth B 321 (2014) 24-29.

[22] Y.B. Miao, B. Beeler, C. Deo, M.I. Baskes, M.A. Okuniewski, J.F. Stubbins, J Nucl Mater 456 (2015) $1-6$.

[23] C. Ronchi, J Nucl Mater 96 (1981) 314-328.

[24] J.Y. Oh, Y.H. Koo, J.S. Cheon, B.H. Lee, D.S. Sohn, J Nucl Mater 372 (2008) 89-93.

[25] J.W. Harrison, J Nucl Mater 31 (1969) 99-\&.

[26] H.X. Xiao, C.S. Long, Chinese Phys B 23 (2014) 1-5.

[27] H.X. Xiao, C.S. Long, X.F. Tian, S.J. Li, Mater Design 74 (2015) 55-60.

[28] W.G. Wolfer, Philos Mag A 58 (1988) 285-297.

[29] P.B. Johnson, K.J. Stevens, R.W. Thomson, Nucl Instrum Meth B 62 (1991) 218-227.

[30] S.Y. Hu, D.E. Burkes, C.A. Lavender, D.J. Senor, W. Setyawan, Z.J. Xu, J Nucl Mater 479 (2016) 202-215.

[31] D.E. Smirnova, A.Y. Kuksin, S.V. Starikov, J Nucl Mater 458 (2015) 304-311.

[32] S. Plimpton, J Comput Phys 117 (1995) 1-19.

[33] W.G. Hoover, Phys Rev A 31 (1985) 1695-1697.

[34] W.G. Hoover, Phys Rev A 34 (1986) 2499-2500.

[35] A. Stukowski, K. Albe, Model Simul Mater Sc 18 (2010) 1-13.

[36] J.D. Honeycutt, H.C. Andersen, J Phys Chem-Us 91 (1987) 4950-4963. 
[37] C.L. Kelchner, S.J. Plimpton, J.C. Hamilton, Phys Rev B 58 (1998) 11085-11088.

[38] A. Stukowski, V.V. Bulatov, A. Arsenlis, Model Simul Mater Sc 20 (2012) 1-16.

[39] J. Rest, G.L. Hofman, Y.S. Kim, J Nucl Mater 385 (2009) 563-571.

[40] A.B. Kaplun, A.B. Meshalkin, High Temp+ 41 (2003) 319-326.

[41] P.B. Johnson, A.L. Malcolm, D.J. Mazey, Nature 329 (1987) 316-318.

[42] W.G. Wolfer, Philos Mag A 59 (1989) 87-103.

[43] J.H. Evans, A. Vanveen, L.M. Caspers, Scripta Metall Mater 17 (1983) 549-553. 


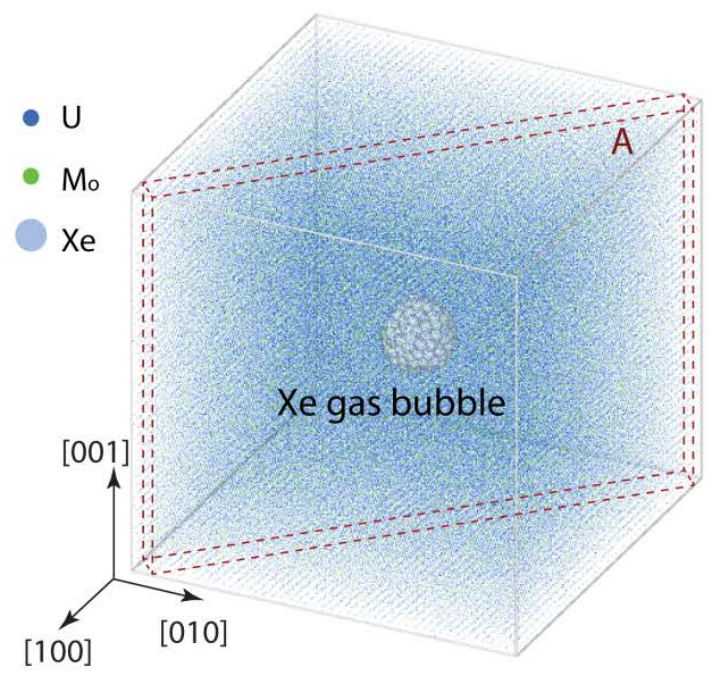

(a)

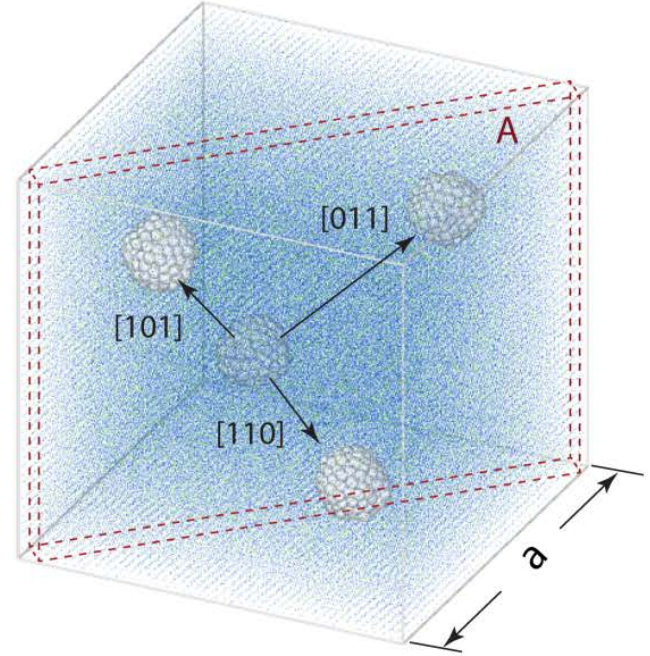

(b)

Figure 1. 

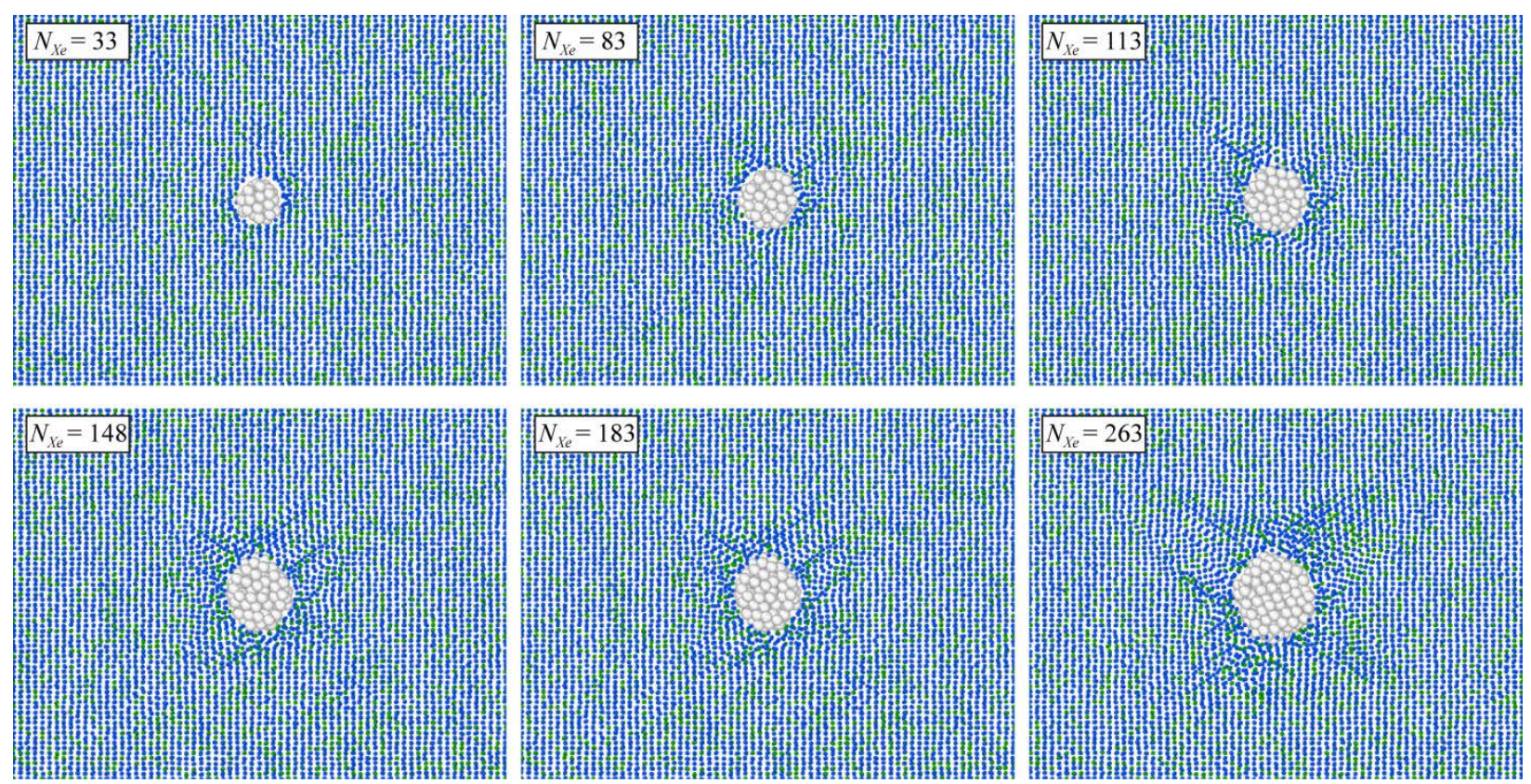

Figure 2. 


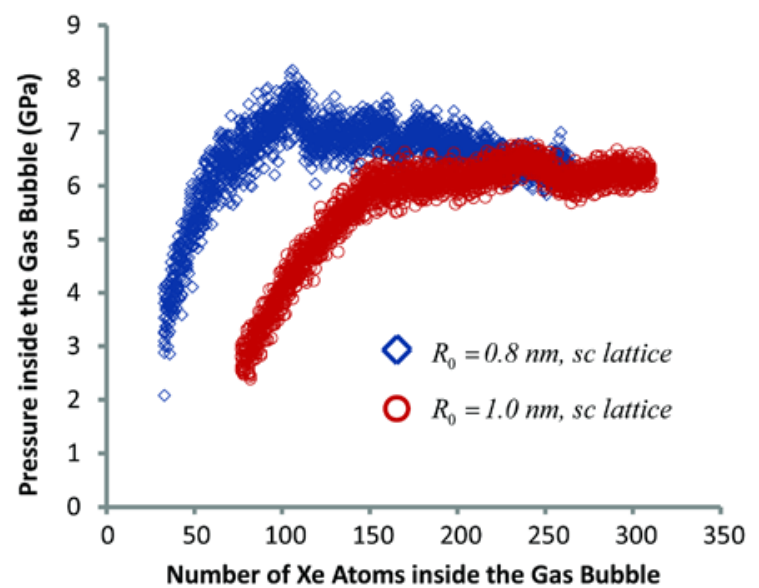

(a)

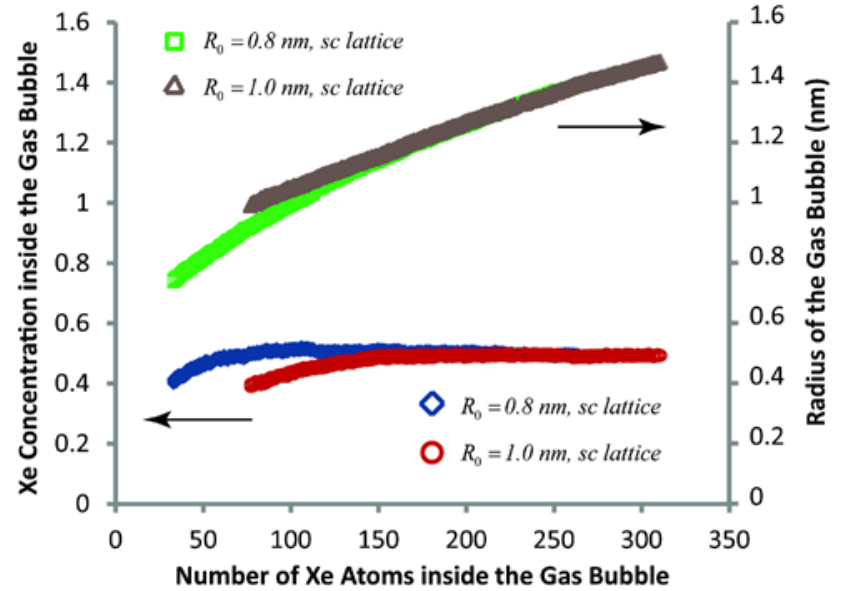

(b)

Figure 3. 


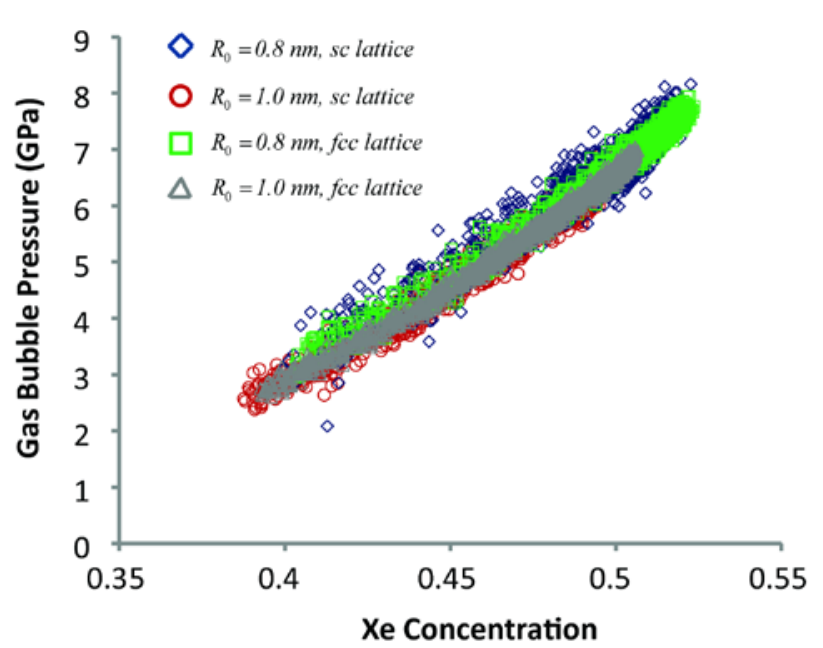

(a)

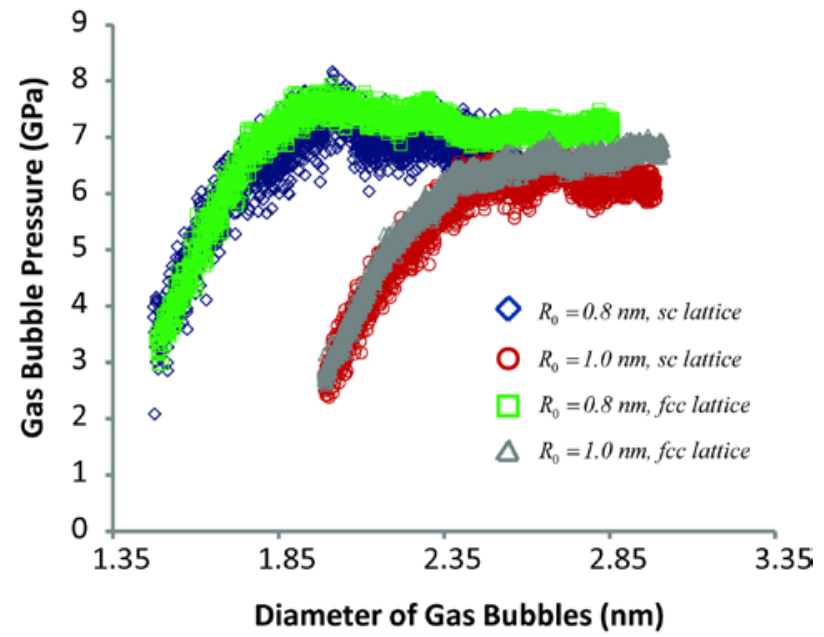

(b)

Figure 4. 


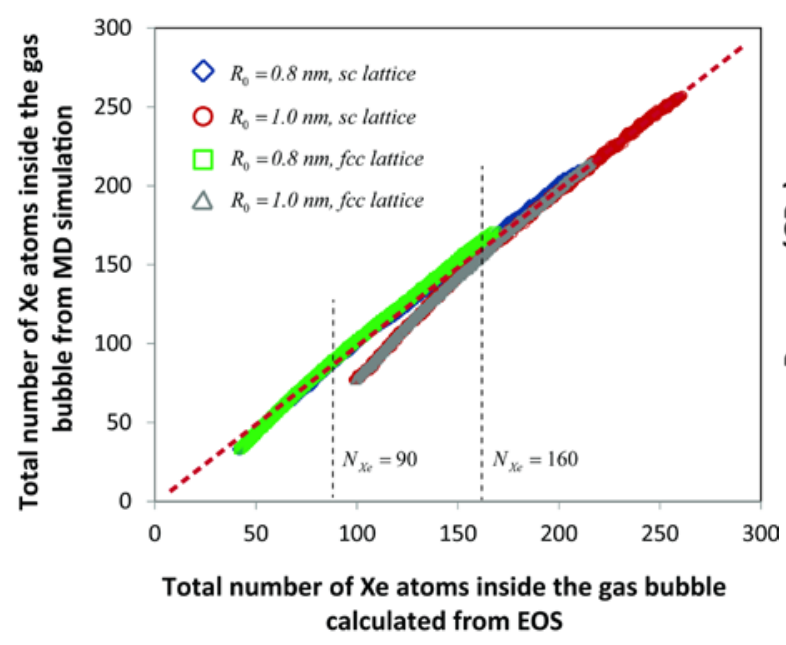

(a)

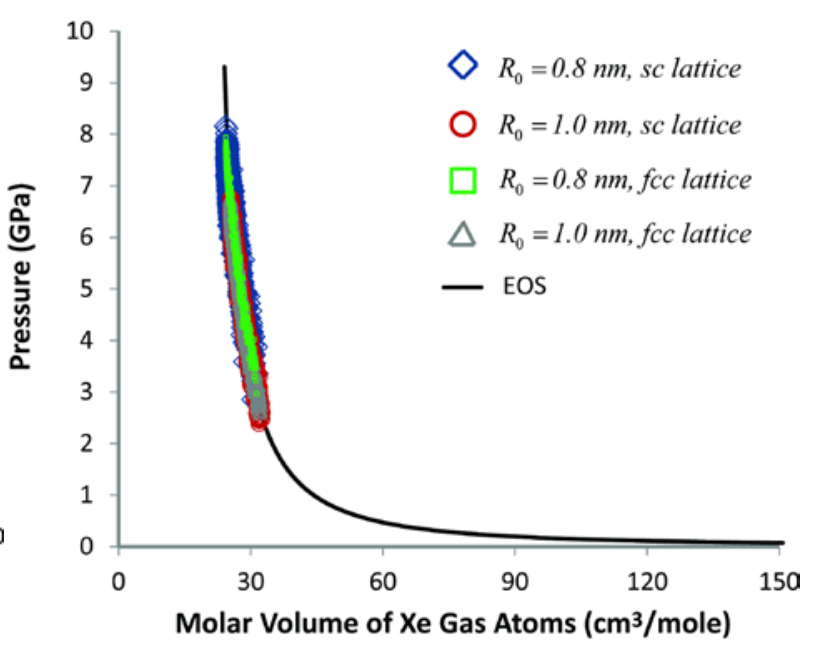

(b)

Figure 5. 

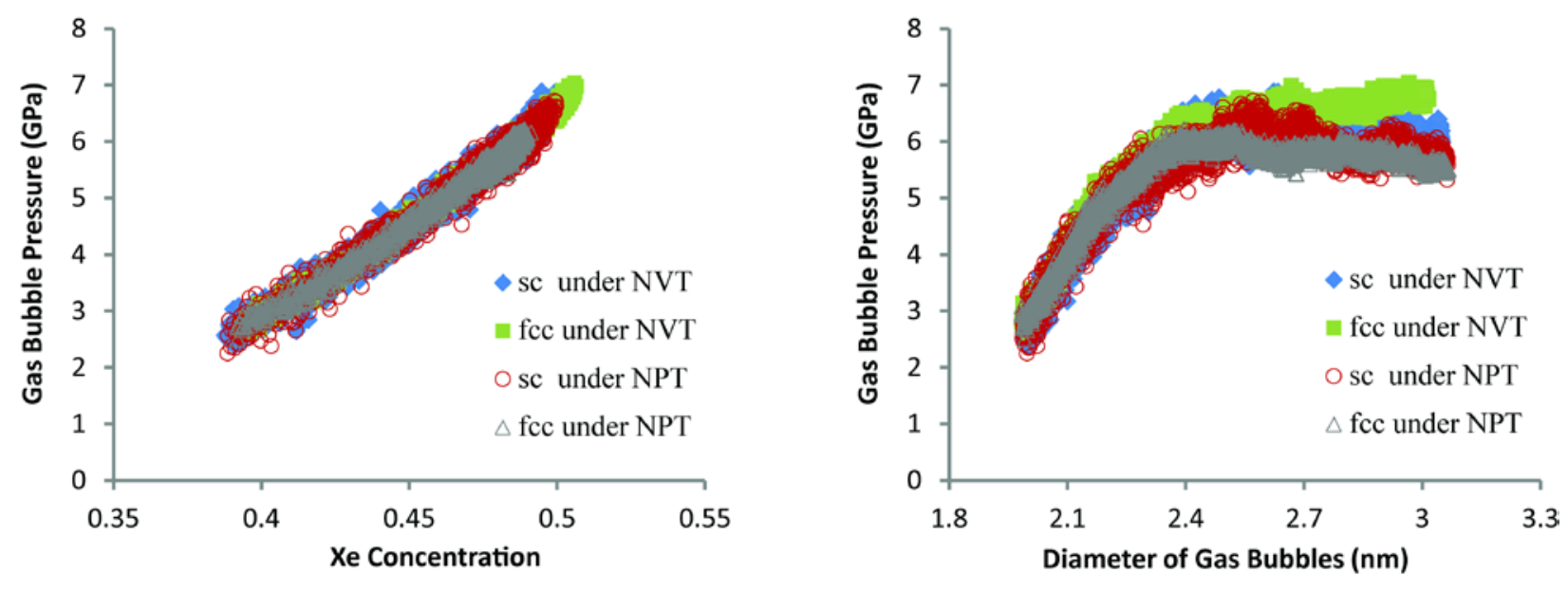

(a) (b)

Figure 6. 


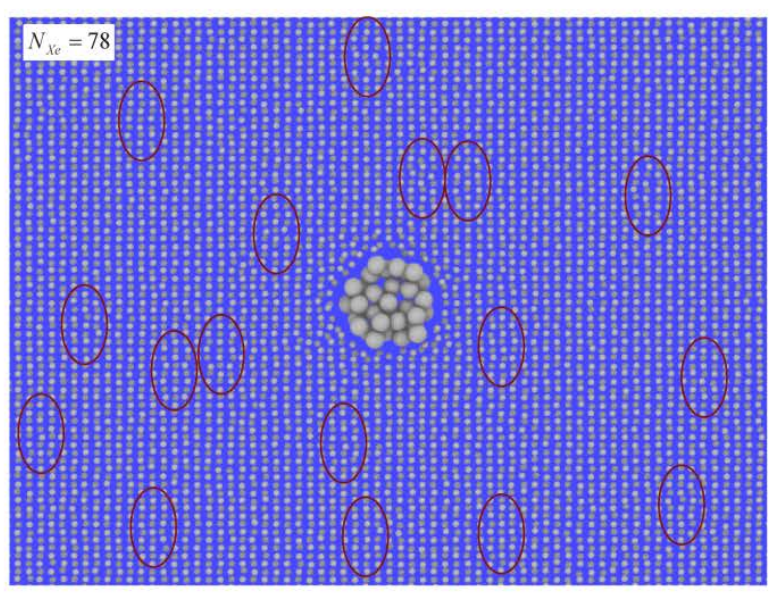

(a)

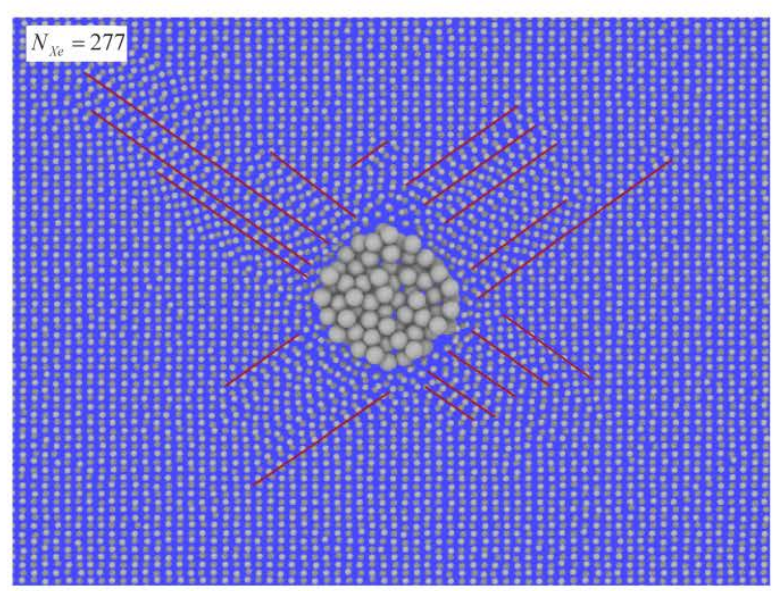

(c)

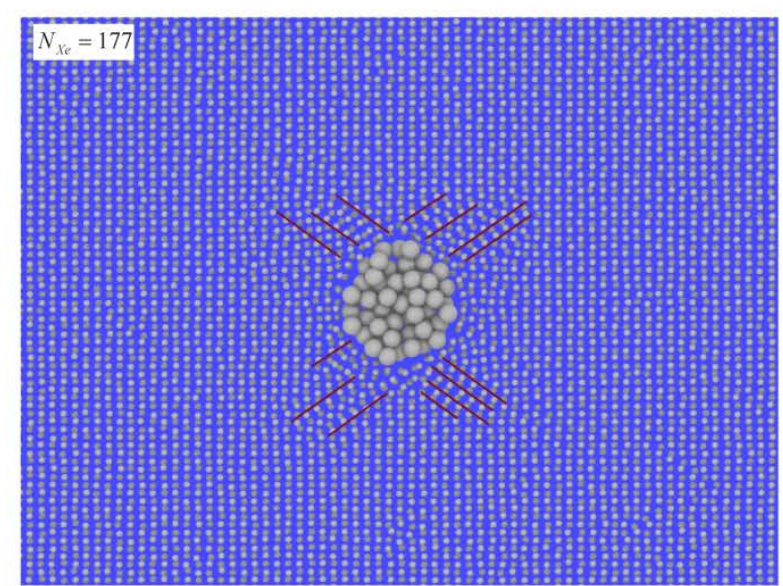

(b)

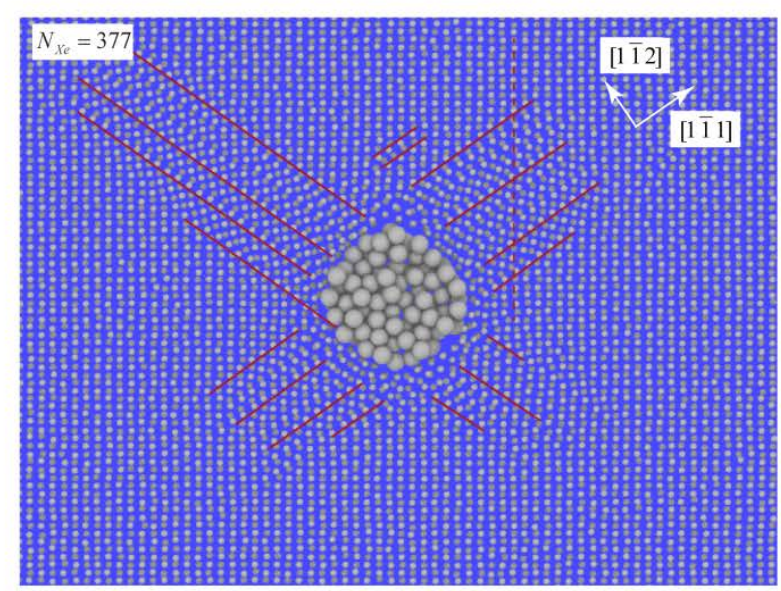

(d)

Figure 7. 


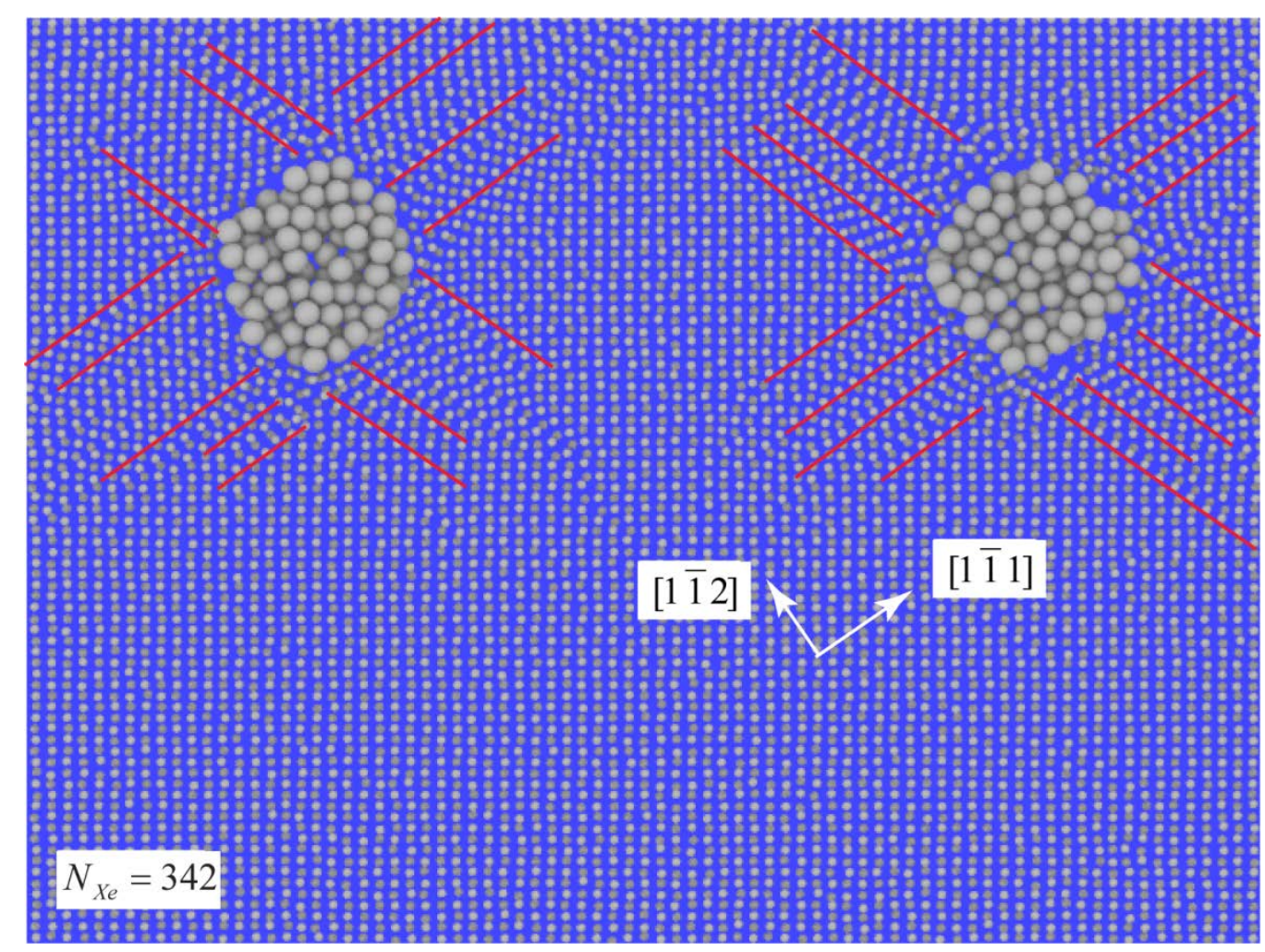

Figure 8. 


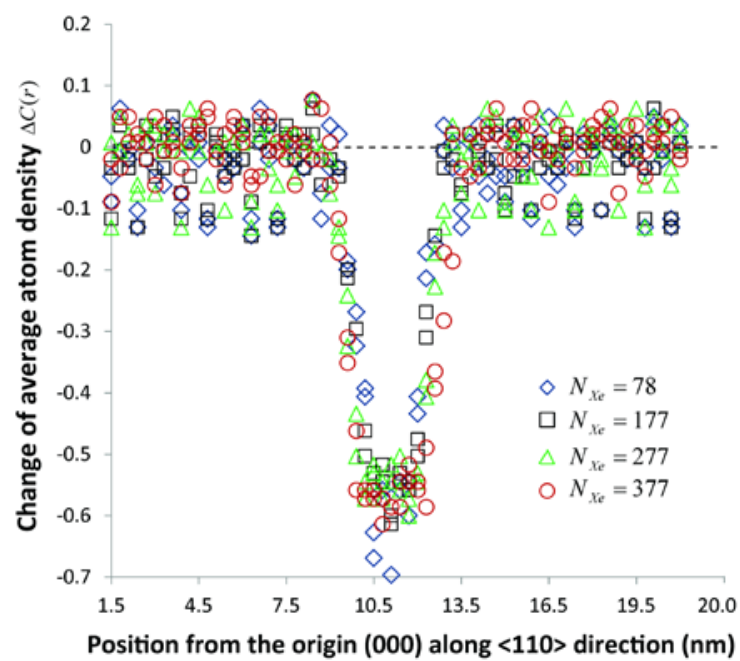

(a)

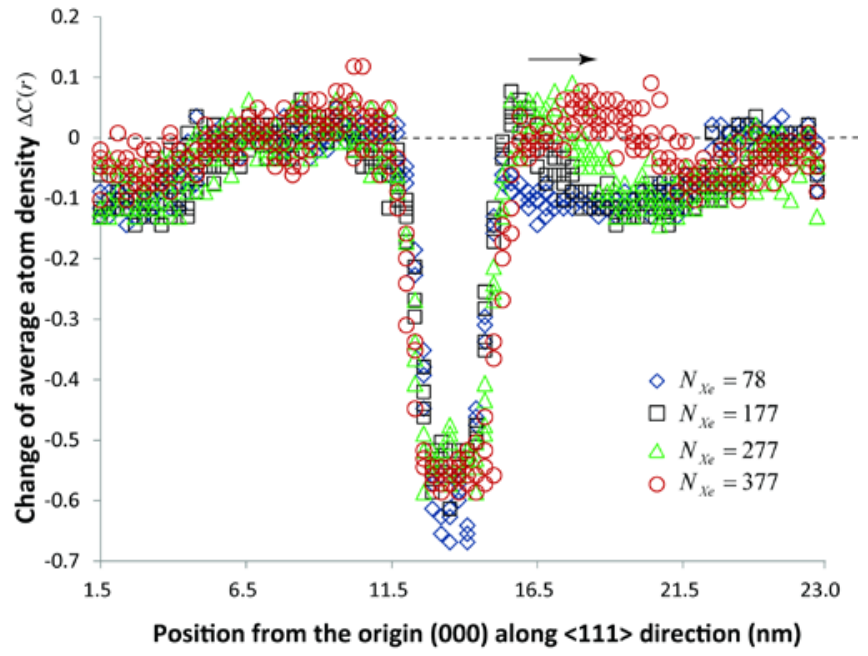

(b)

Figure 9. 


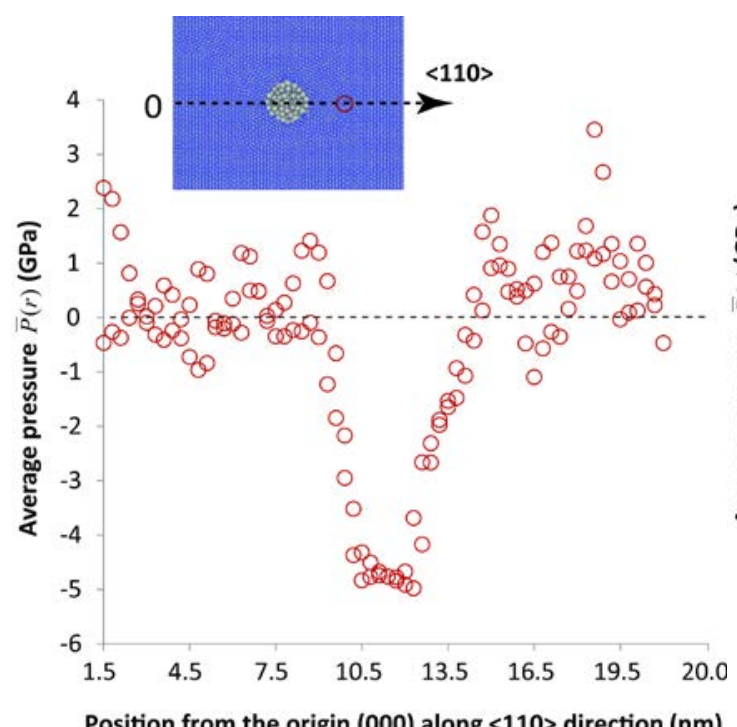

Position from the origin (000) along $<110>$ direction $(\mathrm{nm})$

(a)

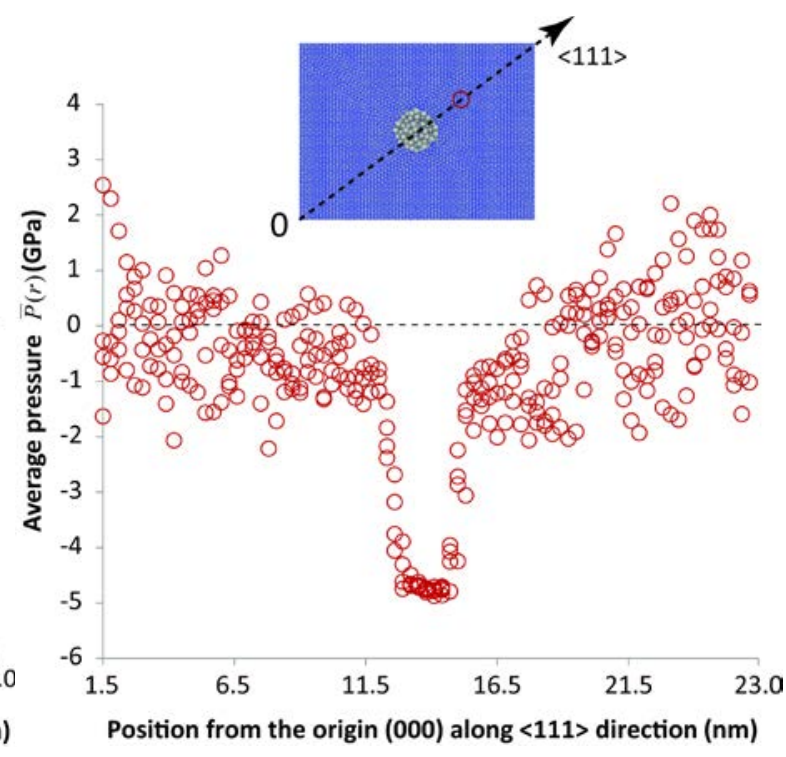

(b)

Figure 10. 